\title{
Trabeculectomy versus trabeculotomy in congenital glaucoma
}

\author{
$S$ C DEBNATH, K D TEICHMANN, AND K SALAMAH
}

From the Department of Ophthalmology, Armed Forces Hospital, PO Box 7897, Riyadh 11159, Saudi Arabia

SUmmary Congenital glaucoma is one of the main causes of blindness in children and is more common in Saudi Arabia than in Western countries, perhaps owing to the prevalence of consanguinity with families. Trabeculotomy has given poorer results in Saudi Arabia than elsewhere. This study compared the results of trabeculotomy (67\% success rate) with trabeculectomy (54\% success rate), and reports fewer complications following trabeculotomy.

Congenital glaucoma occurs in 1:10000 births in Western societies ${ }^{1-4}$ and is commoner in Saudi Arabia, with a reported frequency of 1:2500. 'Among all ophthalmic patients in the Western countries this accounts for $0 \cdot 1-0.4 \%$ of all conditions and in institutions for the blind; $2-15 \%$ of patients have congenital glaucoma. ${ }^{\text {In }}$ Saudi Arabia it accounts for $16 \%$ of all blindness in children."

Most cases appear to be sporadic, but $10 \%$ are familial. Most of these cases show transmission by an autosomal recessive trait, but dominant types have been described. It seems likely that in Saudi Arabia most cases are inherited owing to the social pattern of marrying within families.?

Various surgical procedures have been described for the management of congenital glaucoma. Goniotomy and trabeculotomy have proved most popular, with the success rate of $80-93 \%$ in Western societies. ${ }^{7-9}$ Trabeculectomy on the other hand has proved less successful, having a variable success rate and more serious complications. ${ }^{10-14}$

There has been no study directly comparing the results of trabeculotomy with trabeculectomy for congenital glaucoma. A retrospective study was therefore made to compare two groups of children treated by each method. There was no obvious selection involved, since one surgeon preferred trabeculotomy, while the other preferred trabeculectomy, with children being referred to one or other without prior knowledge of the surgery intended.

The success of the procedure was measured by reduction of the intraocular pressure to, or below, 16 $\mathrm{mmHg}$ as measured by applanation tonometry under general anaesthesia and without additional medicaCorrespondence to S C Debnath, FRCS. tion. Normal values for intraocular pressure were based on a previous study in Saudi children under general anaesthesia, ${ }^{15}$ in which 33 consecutive nonglaucomatous children were examined, from birth to 6 years of age, who were having a variety of other ophthalmic surgical procedures such as probing and syringing of the nasolacrimal duct, surgery for lid chalazion, strabismus, ptosis, and lens aspiration.

The mean intraocular pressure by hand held Perkins tonometer was 10.2 (range $6-15) \mathrm{mmHg}$ and by Schiøtz tonometer 12.5 (range 5.9-20.6) $\mathrm{mmHg}$. The distributions of these values were calculated to give valid reference ranges of $4-16 \mathrm{mmHg}$ for the applantation tonometer and $3-22 \mathrm{mmHg}$ for the Schiøtz tonometer. ${ }^{15}$

\section{Patients and methods}

The retrospective study covered all cases of congenital glaucoma treated at Riyadh Armed Forces Hospital from January 1981 to April 1988. Each patient's age, sex, family history, and consanguinity history were noted, and each underwent a careful ocular examination under general anaesthetic (halothane, nitrous oxide, and oxygen). The patient's corneal diameter, corneal integrity, and intraocular pressure (IOP) measurement both with Perkins hand held tonometer and then Schiøtz tonometer were recorded. In addition retinoscopy, gonioscopy, and funduscopy were performed when the media was clear. Children with proved congenital glaucoma underwent either trabeculectomy or trabeculotomy under general anaesthesia according to the surgeon's choice irrespective of the IOP recorded. 
The surgical technique used for trabeculectomy was basically that of Cairns. ${ }^{16}$ A limbal-based conjunctival flap was prepared in the superior quadrant. Underlying Tenon's capsule was then dissected from episclera and excised generously. A thin, rectangular, partial-thickness scleral flap measuring approximately $4 \times 4 \mathrm{~mm}$ was prepared by dissecting well forward into clear cornea. A $2 \times 2 \mathrm{~mm}$ section of deep limbal tissue was excised from beneath the base of the rectangular flap. A small peripheral iridectomy was then performed beneath the fistula. The scleral flap was approximated with two 10-0 nylon sutures, and the conjunctival incision was closed with a tight running suture of 8-0 Vicryl. The eye was dressed with mydriatics and an antibiotic-corticosteroid ointment at the end of the procedure.

The surgical technique followed for trabeculotomy ab externo was that of McPherson. ${ }^{17}$ A limbal-based conjunctival flap was fashioned in the superior quadrant of the operated eye. A thin limbal-based partial thickness scleral flap measuring approximately $4 \times 2 \mathrm{~mm}$ was dissected down to the corneoscleral limbus. A $2 \mathrm{~mm}$ radial incision was then made at the corneoscleral transition zone, where the Schlemm's canal lay. To confirm the canal's identity a 5-0 nylon suture was threaded through the cut edge. The exposed portion of suture was then bent up and down, it being noted whether it returned to a position parallel to the canal when released. Its failure to do so suggested that a false passage may have been created into the anterior chamber or supraciliary space.

One arm of a McPherson's trabeculotome was then threaded into Schlemm's canal with the other parallel arm as a guide. The handle was then rotated, so that the arm within the canal tore through the trabecular meshwork into the anterior chamber. The same procedure was then performed on the other side of the radial incision. The scleral and conjunctival incision was then closed with 10-0 nylon and 8-0 Vicryl suture respectively. Mydriatics and antibioticcorticosteroid ointment was used for dressing at the end of the procedure.

Interoperative and postoperative complications were recorded, and the children were followed up at regular intervals by examining them under general anaesthesia with the same anaesthetist whenever possible. Each examination included recording the corneal diameter, corneal integrity, intraocular pressure level, results of retinoscopy, and disc pathology.

\section{Results}

Between January 1981 and April 1988, 42 Saudi children with congenital glaucoma were treated. Ten patients were excluded from the study either because of short follow up, multiple operations, or partial treatment in other hospitals. There were 18 male and 14 female children. Twenty nine presented with bilateral and three with unilateral disease. A history of consaguinity among the parents was present in 22 out of 32 patients. Sixty one eyes (32 patients) underwent surgery. Thirty eyes (16 patients) underwent trabeculectomy and the other 31 eyes (16 patients) underwent trabeculotomy. Only four patients had trabeculectomy in one eye and trabeculotomy in the fellow eye; otherwise either trabeculectomy or trabeculotomy was performed in both eyes except in unilateral cases. There were 19 eyes (10 in the trabeculectomy group and 9 in the trabeculotomy group) which presented with a corneal diameter greater than $14 \mathrm{~mm}$. Ten patients (15 eyes trabeculectomy and 5 eyes trabeculotomy) presented with an initial IOP greater than $35 \mathrm{mmHg}$. Twenty eight patients (15 trabeculectomy and 13 trabeculotomy) presented with disease either at birth or soon after. The mean IOP by applantation tonometry was $35 \mathrm{~mm} \mathrm{Hg}$, range 23-55 $\mathrm{mmHg}$. The mean follow-up period was $11 \cdot 2$ months (range 3-30 months). Sixteen eyes (54\%) were successfully treated in the trabeculectomy group compared with 21 eyes $(67 \%)$ in the trabeculotomy group. This difference in success rate between the two groups was not statistically significant.

More serious complications such as loss of vitreous, endophthalmitis, and shallow anterior chamber occurred in the trabeculectomy group. Hyphaema was more common in the trabeculotomy group, but was benign and caused no additional problems (Table 1).

\section{Discussion}

It is our impression that congenital glaucoma is not uncommon in Saudi Arabia, while it is rare in the

Table 1 Surgical complications

\begin{tabular}{llllll}
\hline Operation & Hyphaema & $\begin{array}{l}\text { Shallow ant. } \\
\text { chamber }\end{array}$ & Lens opacity & $\begin{array}{l}\text { Posterior } \\
\text { synaechiae }\end{array}$ & Vitreous loss \\
\hline Trabeculectomy & 1 & 1 & 1 & 2 & 3 \\
Trabeculotomy & 11 & 0 & 0 & 0 & 0 \\
\hline
\end{tabular}


Western world. A history of consanguinity of the parents was present in $22(68 \%)$ out of 32 patients in our study group, which suggests that the inherited form is more common in the Kingdom of Saudi Arabia owing to high consanguinity among Saudi couples than in the Western countries. Sixteen patients ( 30 eyes) underwent trabeculectomy and the other 16 patients (31 eyes) underwent trabeculotomy. Goniotomy and trabeculotomy have been shown to have a high success rate $(80-93 \%)$ for congenital glaucoma in the Western world. ${ }^{7-9}$ However, our success rate with trabeculotomy was only $67 \%$ which is comparable to the success rate obtained by Kazi et al. in a similar group of patients treated at King Khaled Eye Specialist Hospital, Saudi Arabia. ${ }^{3}$ It appears that congenital glaucoma in Saudi Arabia does not respond so well to trabeculotomy as in the Western world. We share the belief of Kazi et al. that the main causes of failure from the trabeculotomy procedure in this Kingdom are: usually late presentation of the patient, cases being present at birth, more inherited cases, and more severe cases with larger corneal diameters, cloudy corneas, and high initial IOP at the time of presentation. ${ }^{3}$

Trabeculectomy for congenital glaucoma has not become popular owing to its variable success rate and higher complication rate. There are several reasons for this. They include anatomical factors such as enlargement, stretching, and thinning of the coats of the eye; deep anterior chambers with subluxated lenses; limbal ectasias and staphylomas; late fibrosis of the iris root and trabecular meshwork; late disappearance of Schlemm's canal and increased thickness of infantile and juvenile Tenon's capsule, ${ }^{111}$ Is complexity of associated pathology, management factors, and the number of previous operations.

Our success rate of $54 \%$ in the trabeculectomy group compares with a $50 \%$ success rate of Beauchamp and Parks,"' but it should be noted that their cut-off level was $24 \mathrm{mmHg}$, while ours was 16 $\mathrm{mmHg}$. If a similar cut-off level is chosen, then our success rate goes up close to $60 \%$. Mehta et al." reported a $66 \cdot 7 \%$ success rate in congenital glaucoma with trabeculectomy, but they studied only three patients. Similarly, Joesph and Livingston ${ }^{12}$ reported a $48 \%$ absolute success without medical treatment and $28 \%$ partial success with medical treatment together with trabeculectomy in a study of 50 cases of congenital glaucoma with cut-off level for the IOP of $20 \mathrm{mmHg}$. Luntz ${ }^{13}$ reported a higher success rate of $95 \%$ with trabeculectomy, but most of his patients were older children and therefore not directly comparable with our patients. Werther ${ }^{14}$ reported a $90 \%$ success rate with trabeculectomy, but his followup period was very short and again was limited to a small number of patients. Generous excision of Tenon's capsule, and partial thickness scleral flap dissection well beyond the limbus into the clear cornea, as recommended by Werther,$^{14}$ may have contributed to our reasonable success rate in trabeculectomy. We are inclined to share the belief of Werther ${ }^{14}$ that increased thickness of Tenon's capsule and the subsequent scarring by it contributes to the failure of the filtering procedure in children.

\section{CONCLUSION}

The success rate of trabeculectomy and trabeculotomy procedures are very close in this study, but we still recommend trabeculotomy as an initial primary procedure because trabeculectomy carries a higher rate of complications. However, trabeculectomy may be an acceptable alternative when microsurgical facilities and specially trained surgeons are not available, as is the case in many developing countries.

The authors thank the Ophthalmic Secretary, Miss Julie Williams. for typing the manuscript.

\section{References}

1 Jaafar MS. Care of the infantile glaucoma patient. Ophthalmology Annual. New York: Raven Press, 1988: 7: 1537.

2 Logan A, Jaafar MS. Infantile glaucoma: an autosomal dominant family. In: Tabbara KF, ed. Second annual symposium on new developments in ophthalmology, 28-31 January 1985. Saudi Arabia: Riyadh, King Khaled Eye Specialist Hospital, 1985: 118-9 (abstr).

3 Kazi GA, Jaafar MS, Traverso CE, Tomey KF. One-year experience of congenital glaucoma at King Khalid Eye Specialist Hospital. In: Tabbara KF, ed. Third annual symposium on new developments in opthalmology, 27-30 January 1986. Saudi Arabia: Riyadh, King Khaled Eye Specialist Hospital, 1986: 253-4 (abstr).

4 Miller SJH. Genetic aspects of Glaucoma. Trans Ophthalmol Soc UK 1962; 81: 425-34.

5 Duke-Elder S. Congenital deformities. System of ophthalmology. St Louis: Mosby, 1969: 3 (2): 548-65.

6 Tabbara KF. Badr IA. Changing pattern of childhood blindness in Saudi Arabia. Br J Ophthalmol 1985; 69: 312-5.

7 DeLuise VP, Anderson DR. Primary infantile glaucoma (congenital glaucoma). Surv Ophthalmol 1983; 28: 1-19.

8 Quigley HA. Childhood glaucoma: results with trabeculotomy and studies of reversible cupping. Ophthalmology 1982: 89: 21925.

9 McPherson SD Jr, McFarland D. External trabeculotomy for developmental glaucoma. Ophthalmology 198(); 87: 302-5.

10 Beauchamp GR, Parks MM. Filtering surgery in children: barriers to success. Ophthalmology 1986; 86: 170-80.

11 Mehta KR, Sathe SM. Karyekar SD. Trabeculectomy ab-externo. Indian J Ophthalmol 1974; 22: 9-12.

12 Joseph A. Trabeculectomy in congenital glaucoma. Indian $J$ Ophthalmol 1981: 29: 81-2.

13 Luntz MH, Livingston DG. Trabeculotomy ab-externo and trabeculectomy in congenital adult onset glaucoma. $A m J$ Ophthalmol 1977: 83: 174-9.

14 Werther DE. Trabeculectomy in congenital glaucoma. Excerpta Medica 1979; 2: 1517-8.

15 Debnath SC, Lambourne A, Vandenberg AA, Laghari NA. Wani AR, Salamah K. Intraocular pressure in Saudi children under general anaesthesia. Saudi Med J 1988: 9: 296-302. 
16 Cairns JE. Trabeculectomy: preliminary report of a new method. Am J Ophthalmol 1968: 66: 673-9.

17 McPherson SD Jr. Results of external trabeculotomy. Am J Ophthalmol 1973; 76: 918-20.
18 Yanoff M, Fine B. Occular pathology. Hagerstown: Harper Row, 1975: 590.

Accepted for publication 28 December 1988. 